\title{
Atomistic insight into novel co-delivery of doxorubicin and paclitaxel using fullerene modified by dimethyl acrylamide trimethyl chitosan: $A$ computational study
}

\section{Ehsan Alimohammadi}

Kerman University of Medical Sciences

Reza Maleki ( $\nabla$ rezamaleki96@gmail.com )

Sharif University of Technology https://orcid.org/0000-0002-5169-5848

Hossein Akbarialiabad

Shiraz University of Medical Sciences

Mohammad Dahri

Shiraz University of Medical Sciences

Research

Keywords: Doxorubicin, Paclitaxel, Molecular Dynamics, Drug Release, Smart Drug Delivery, Fullerene

Posted Date: October 12th, 2020

DOl: https://doi.org/10.21203/rs.3.rs-21545/v2

License: (c) (i) This work is licensed under a Creative Commons Attribution 4.0 International License.

Read Full License 


\section{Abstract}

Background: The distribution of drugs could not be controlled in the conventional drug delivery system. This has led to the development of a smart nanoparticle-based drug delivery system, known as smart drug delivery systems. In cancer research, novel biocompatible nanocarriers have received much attention for various ranges of anticancer drugs.

In the current study, the effect of a novel co-polymer "dimethyl acrylamide-trimethyl chitosan" was investigated on drug delivery of doxorubicin and paclitaxel utilizing modified fullerene nanocarrier.

This study was performed via molecular dynamics simulation based on acidic $\mathrm{pH}$ sensitivity of the cancer microenvironment. Furthermore, hydrogen bonds, diffusion coefficient, gyration radius, and drugcarrier interaction energies were investigated here. Interestingly, a simultaneous $\mathrm{pH}$ and temperaturesensitive system was proposed for paclitaxel and doxorubicin for a co-polymer. A pH-sensitive and temperature-sensitive copolymer was used based on trimethyl chitosan and dimethyl acrylamide, respectively. In such a dualistic approach, co-polymer makes an excellent option to have two properties in one bio-polymer.

Results: The results suggest the dramatic and indisputable role of the co-polymer in the release of doxorubicin and paclitaxel in cancerous tissues, as well as an increased biocompatibility and drug uptake in a neutral state. The validation test was performed by repeated simulations of a similar article. The results are very close to those of the reference paper.

Conclusions: Overall, conjugated fullerene and dimethyl acrylamide-trimethyl chitosan (DMAA-TMC) as carriers can be a good proposition for loading, delivery, and release of anti-cancer drugs based on a $\mathrm{pH} /$ temperature-sensitive smart drug delivery system.

\section{Introduction}

Multiple factors are involved in cancer initiation and progression (1). These factors can be intrinsic, such as susceptibility to Chronic myelogenous leukemia (CML) in a newborn with Philadelphia Chromosome or extrinsic,includingtobacco smoking, diet, infectious disease, ion and non-ion radiation, and so on. Through complex mechanisms, these factors will promote the expression of oncogenes (e.g. MDM2 and $\mathrm{BCL}$ ) or suppresses the tumor inhibitor genes (genome guardians) such as P-53 and RB (2-9). Nowadays, various treatment methods including chemotherapy, radiotherapy, and target-therapy are common ways of cancer therapy,. each of which has specific pros and cons. Researchers are focusing on optimizing the drug's efficacy while reducing undesired side effects (10). In the current century, nanobiotechnology has gained ground in cancer treatment and and diagnosis $(11,12)$. Development in targeted nano-drug delivery has resulted in the progression of the smart drug delivery concept (13). The maximum level of optimization, a surge in the therapeutic index and drug concentration in affected tissue,and reduced damage to healthy cells will be achieved in such a system $(14,15)$. 
Doxorubicin (DOX) and Paclitaxel (PAX) are two crucial common combination anticancer agents in cancer therapy. Previous Simultaneous administration studies suggested that DOX/PAX combination could be effective in metastatic cancer, especially in breast cancer. Due to synergism, this combination is also effective in inhibition of multidrug resistance (MDR) cancer cells (16). DOX, as an anthracycline, interferes with the production of proteins that are essential for cancer cell proliferation and impede cancer growth (17-19). It seems that this drug acts via binding to DNA and subsequent inhibition of nucleic acid production by disrupting the molecular structure and further steric hindrance (20). As with any anticancerdrug, one of the undesirable side effects can be damaging surrounding cells of the tumor (21). As mentioned above, targeted drug delivery can minimize the impacts of this drug on non-cancerous cells of the body and maximize the drug efficiency for cancer treatment $(18,22,23)$. PAX is a novel antimicrotubular agent that inhibits the production of microtubules from tubulin dimers and stabilizes the microtubules by preventing depolymerization $(23,24)$. This stabilization inhibits the dynamic identification of the microtubule network, which is required for critical interphase and mitotic cellular functions $(25,26)$. PAX is one of the most effective drugs in the treatment of breast cancer with chemotherapy. Also, utilizing nanocarriers to optimize PAX's efficacy seems to be promising (27).

Nano-based Drug delivery systems have an essential role in the development of chemotherapeutic agents, cancer drug targeting, the selective antiproliferative effect (28), and minimizing adverse drug sideeffects (29). Multiple nanoparticles are capable carriers in enhancing the delivery of DOX/PAX; these agents are solid lipid nanoparticles (30). Among these, 60-carbon fullerenes are one of the compounds that have recently received much attention in DOX/PAX drug co-delivery is (31). The 60-carbon fullerenes (C60) are about 1 nanometer in diameter, nearly half the width of a DNA helix; it has a small size and spherical shape, enables it to conveniently cross biological membranes and barriers and reach the cell (32). By having extended surfaces area, the surface properties of these particles can be readily modified and functionalized using functional groups and compound. The functionalization of these particles will increase their solubility, biocompatibility, and potential to deliver various materials within the body. These particles can be used as carriers of biological molecules such as protein, DNA, and drugs. Drug compounds can be loaded onto or within these structures (33). Another interesting issue in drug delivery is co-targeting and co-transportation in which two or more compounds are targeted and transported. Many surveys have studied the potential of fullerene (34). The particular physical and chemical features of $\mathrm{C60}$ as a nanocarrier for anticancer agents for drug delivery include size, triangular shape, surface charge, surface chemistry, hydrophobicity, loading's potential, and especially the ability to cross various biological barriers in vivo without inducing an immune response.

C60 can bond with specific cell receptors and intracellular target molecules for targeted delivery of therapeutic agents (35). Research has shown that C60 enter the cell vertically through mediated endocytosis mechanism because of there similarity to clathrin. The drug can be encapsulated by the C60 and protected during circulation through the body. After reaching the target site, the encapsulating materials will be degraded after the drug release from the C60. The encapsulated drug should be proportional to the diameter and size of the C60 (36). Despite their specific inherent properties, relevant 
concerns have been mentioned regarding the toxicity of $\mathrm{C} 60$, as several studies have manifested that pristine $\mathrm{C} 60$ can instigate biological destruction (36).

In this work, $\mathrm{C} 60$ bioconjugation with a novel biodegradable and biocompatible polymer dispels its biological concerns and converts it to a safer, more reliable nanocarrier. On the other hand, many investigations indicated that the $\mathrm{C} 60$ functionalized by a carboxylic group rendered it pharmacodynamically and pharmacokinetically better for drug delivery. Overall, the pH-sensitive C60 modified with non-bonded interaction of dimethyl acryl amide-trimethyl chitosan (DMAA-TMC) in this work produces a biocompatible $\mathrm{C} 60$ that can capably be nominated as smart and target delivery systems. DMAA-TMC, as a modified polysaccharide from chitosan, plays a vital role in adsorption enhancement of novel macromolecule delivery systems. In previous studies, the role of chitosan and acrylamide polymers was clarified separately in the improvement of drug delivery systems. In our current study, the combination of two polymers DMAA and TMC, in sillico by $\mathrm{C} 60$ carrier was investigated for the first time. Moreover, it's non-bonded interaction with $\mathrm{C} 60$ and positive surface charge induce the specific properties in drug adsorption and drug release. Because of pores in DMAA-TMC, modified C60 was evaluated as a potential tool to improve the loading and co-release of DOX/PAX in physiological and cancerous $\mathrm{pH}$, respectively (37).

Molecular dynamics is a powerful tool that can provide qualitative and quantitative information on the physicochemical interactions and mechanisms of pharmaceutical systems. In the study of molecular dynamics, a system is first considered that consists of " $\mathrm{N}$ " particles inside a box called "computational box". The location and velocity of the particles at each step can be used to calculate all the static and dynamic properties of the system. From the theorists' point of view, the importance of molecular dynamics studies is that they provides accurate quasi-experimental results for a well-defined model. Molecular dynamics serves somewhere between laboratory experiments and theory and is considered a virtual analysis and provides better mechanistic understanding from in comparion to machine learning methods(38-40).

Empirical experiments are highly important but may impose a significant financial burden on researchers. Hence, there are many studies today on molecular dynamics to simulate drug delivery systems in cancer. However, therefore, the C60s as an attractive carrirer for drug release were used to compare the uptake, diffusion, and release of DOX and PAX from C60 in the presence of chitosan polymer. Given the unique properties of $\mathrm{C} 60$, this could be an excellent introduction to the wider use of carbon $\mathrm{C} 60$ in the loading and release of anticancer drugs $(41,42)$.

\section{Methods}

\section{Molecular dynamics simulation}

Gromacs 5.1.2 software was used to perform the simulation; the input structures were prepared with the OPLS-aa force field. Using ACPYPE script, the parameters of the molecule that were converted to Gromax format. All the particles were placed inside the box, and the tip3p water model was used as the 
solvent (43). Energy reduction was performed on all 50,000-step simulation systems by the steepest descent method to eliminate van der Waals interactions and to form hydrogen bonds between water molecules and other molecules. In the next step, the system temperature gradually increased from 0 to $310 \mathrm{~K}$ for 100 picoseconds in constant volume, using the Nose-Hoover algorithm (44).

Moreover, the temperature system coupling rate of $0.5 \mathrm{ps}$ used and then at the constant pressure was equilibrated for $200 \mathrm{ps}$. The Parrinello-Rahman algorithm was used to balance the system pressure. Molecular dynamics simulation was performed at $37^{\circ} \mathrm{C}$ for $50 \mathrm{~ns}$. The cut-off distance was set at 1.2 Particle mesh Ewald (45) was used to calculate the electrostatic force. The linear constraint solver algorithm was used to maintain the length of all links. In order to increase computational speed, the SHAKE algorithm was used to limit the hydrogen atom's bonds (46).

\section{Carbon nanostructures parameters}

The carbon atom charge in these nanostructures was assumed based on the use of naphthalene structure in the zero oplsaa force field. The types of bonds between carbon atoms were defined based on amino acids phenylalanine (47), tyrosine (48), and tryptophan (Trp) (49). The angle type is also determined based on the angles of the aromatic amino acid phenylalanine ring. The charge and the parameters of the functional groups on this nanostructure were defined using a similar structure existing in the oplsaa force field. Lenard-Jones models and Colombian potentials were used to calculate nonbonding interactions such as electrostatic and van der Waals, respectively.

\section{Results And Discussion}

To predict the co-adsorption of DOX/PTX on the DMAA-TMC functionalized C60, utilizing various computer-based mechanisms, co-release, and aggregation of drugs were investigated in cancerous and physiological conditions with pHs 5.5 and 7.4 , respectively.

\section{Drug-C60 Accumulation}

Gyration radius $(\mathrm{Rg})$ is a factor that enables us to analyze the aggregation and stability of molecules such as polymers and resizing of biological macromolecules such as proteins over time (49). The average of the gyration radius at initial and final time is shown in Table 1 and 2 . As shown in Table 1, the gyration radius indicates the accumulation of molecules in one region. The low $\mathrm{Rg}$ indicated a high accumulation in the location. The Gyration radius of DOX and PAX is about $3 \mathrm{~nm}$, indicating the aggregation radius of drug accumulation on the $\mathrm{C} 60$ surface. Due to the dimensions of $\mathrm{C} 60$ and simulation boxes, a useful aggregation of drugs is formed around the $\mathrm{C60}$. This revealed that the polymer molecules are clustered together in this simulation. PAX also has a lower radius than DOX, indicating a better accumulation of DOX in comparison to PAX. Complexation due to the accumulation of PAX molecules is more stable and concentrated. The interaction of hydrophilic polymer DMAA with water molecules and $\mathrm{C} 60$ helps to coat the nanocarrier better in bloodstream, which can improve the 
hydrophilicity of PAX. According to Table 1 and 2 the accumulation of the same drugs is similar in two different pHs states at initial time.

On the other hand, the higher the gyration radius, the greater dispersion between the particles. As shown in Table 2, At acidic $\mathrm{pH}$ the $\mathrm{Rg}$ increase, the stablitly and aggregation of systems decrease, and the system disassembled. Hence, the release of drugs facilated at acidic envirment

\section{Table 1: Neutral pH}

\begin{tabular}{|l|l|l|l|}
\hline Structure & Doxorubicin & Paclitaxel & DMAA-TMC \\
\hline Initial gyration radius & $3.34 \mathrm{~nm}$ & $3.46 \mathrm{~nm}$ & $3.61 \mathrm{~nm}$ \\
\hline Final gyration radius & $2.08 \mathrm{~nm}$ & $2.43 \mathrm{~nm}$ & $2.67 \mathrm{~nm}$ \\
\hline
\end{tabular}

\section{Table 2: Acidic pH}

\begin{tabular}{|l|l|l|l|}
\hline Structure & Doxorubicin & Paclitaxel & DMAA-TMC \\
\hline Initial gyration radius & $3.46 \mathrm{~nm}$ & $3.57 \mathrm{~nm}$ & $3.72 \mathrm{~nm}$ \\
\hline Final gyration radius & $3.74 \mathrm{~nm}$ & $3.69 \mathrm{~nm}$ & $3.91 \mathrm{~nm}$ \\
\hline
\end{tabular}

\section{Drug-nanocarrier interaction}

Hydrogen bonding between two atoms is defined as a donor-acceptor pair with an angle between them less than 30 degrees. The Fig. 1 show changes in the numbers of hydrogen bonds over time between polymer-polymer and polymer-drug and C60-drug for all three simulations are shown in Fig.1. Hydrogen bonding can serve as a hydrophilicity indicator of the carrier. Besides, hydrogen bonding is part of the interatomic forces that can contribute to carrier strength and stability. The analysis of the diagrams below shows that DOX is not bounded to C60s but has many hydrogen bonds with DMAA-TMC. This chart illustrates the crucial role of DMAA-TMC in this drug delivery system. DMAA-TMC interacts and bonds with $\mathrm{C60}$, which makes it more hydrophilic. C60s and DOX, as well as PAX, are hydrophobic compounds, and this is a significant drawback for drug carriers; Because hydrophobic compounds aggregate in water and compose large particles disrupting drug delivery and block the bloodstream.

Furthermore, DMAA-TMC has solved this problem by hydrophilic the complex. The graphs also show that PAX did not form hydrogen bonds with C60s, while PAX makes many hydrogen bonds with DMAA-TMC, which is an essential factor in drug delivery. A comparison between PAX and DOX Shows that the DOXDMAA-TMC hydrogen bonds are more robust than the PAX-DMAA-TMC hydrogen bonds. Therefore, the addition of DMAA-TMC also contributes to better adsorption of DOX as the hydrogen bonds between DOX and DMAA-TMC are relatively stable. 
Fig 1. Interaction hydrogen bonds
a) The number of hydrogen bonds between DOX and $\mathrm{C} 60$ versus time in neutral $\mathrm{pH}$
b) The number of hydrogen bonds between DOX and DMAA-TMC versus time in neutral pH
c) The number of hydrogen bonds between PAX and $\mathrm{C} 60$ versus time in neutral $\mathrm{pH}$
d) The number of hydrogen bonds between DMAA-TMC and PAX versus time in neutral $\mathrm{pH}$
e) The number of hydrogen bonds between DOX and $\mathrm{C} 60$ versus time in acidic $\mathrm{pH}$
f) The number of hydrogen bonds between DOX and DMAA-TMC versus time in acidic $\mathrm{pH}$
g) The number of hydrogen bonds between PAX and C60 versus time in acidic $\mathrm{pH}$
h) The number of hydrogen bonds between PAX and DMAA-TMC versus time in acidic $\mathrm{pH}$

\section{Energy interaction of drug and nanocarrier}

\section{Neutral state; $\mathrm{pH}=7.4$}

In the Fig. 2 and Fig.3, the interaction of the DOX molecule with $\mathrm{C} 60$ is investigated. At neutral pH, electrostatic energy plays a significant part in the total interaction energy. While in the acidic state, the electrostatic energy is zeroed, and the van der Waals energy has a considerable share of the total interaction energy. That is due to the surface charge of the carboxyl functional groups at the $\mathrm{C60}$ surface. The carboxyl group has a negative charge at neutral $\mathrm{pH}$ and no charge at acidic $\mathrm{pH}$.

On the other hand, DOX has a positive charge at neutral $\mathrm{pH}$ and acidic $\mathrm{pH}$. As a result, in the neutral state, drug, and $\mathrm{C} 60$ functional groups, have anonymous and robust electrostatic interactions. The higher the electrostatic energy at neutral $\mathrm{pH}$, the higher the adsorption of the drug onto the nanocarrier surface at this $\mathrm{pH}$. The critical point is that the drug at a neutral $\mathrm{pH}$, which is the $\mathrm{pH}$ of the blood, can transfer satisfactorily to the surface of the C60. and C60, by having a strong attraction to DOX, can serve as an excellent carrier for the drug.

Fig. 2 shows the interaction between DOX and DMAA-TMC, as can be seen in figure c., the van der Waals interaction is close to zero. Still, there is a significant negative electrostatic interaction between the drug and the polymer. This interaction is due to the positive charge of DOX and the negative charge of the polymer at this $\mathrm{pH}$. The negative electrostatic energy indicates a strong attraction between the drug and the DMAA-TMC. DMAA-TMC is an essential aid in the absorption of the drug.

The part b. in Fig. 2 is the energy interaction of PAX with C60. As shown in the figure at neutral pH, the electrostatic energy shows a more significant number, while the energy van der Waals energy is close to zero. $\mathrm{C60}$ is functionalized with carboxyl functional groups. The carboxyl group has a negative charge at neutral $\mathrm{pH}$ and no charge at acidic $\mathrm{pH}$. On the other hand, PAX has zero charges at neutral pH. As a result, 
in the neutral state, the electrostatic energy between PAX and the C60 is close to zero. Van der Waals Energy plays a significant part in the adsorption of PAX onto $\mathrm{C60}$. The following diagram illustrates the energy interaction between PAX and DMAA-TMC at d. As shown in Fig.2, at neutral pH, van der Waals energy shows a more significant number, and electrostatic energy is nearby to zero. PAX has zero charges at neutral $\mathrm{pH}$. As a result, in the neutral state, the electrostatic energy between PAX and DMAA-TMC is close to zero. van der Waals energy plays a significant role in the absorption of PAX onto DMAA-TMC.

\section{b. Cancerous state $\mathrm{pH}=5.5$}

In Fig.4, the interaction energy of the doxorubicin molecule with $\mathrm{C60}$ is investigated. As shown in the figure at acidic $\mathrm{pH}$, the electrostatic energy is shallow and close to zero. At acidic $\mathrm{pH}$, electrostatic energy is zero, and van der Waals energy has a significant contribution to the total interaction energy. This is due to the surface charge of the carboxyl functional groups at the $\mathrm{C60}$ surface. On the other hand, DOX has a positive charge at neutral $\mathrm{pH}$ and acidic $\mathrm{pH}$. As a result, in the neutral state, $\mathrm{C60}$ and drug functional groups have nameless charges and find durable electrostatic interactions. Though, at acidic pHs since C60 and carboxyl group charge becomes zero, the electrostatic interaction energy between C60 and DOX is also zero.

Fig.4.b. Shows the van der Waals and electrostatic energies of PAX and C60. As is clear from the figure, the electrostatic energy is close to zero, and the total energy is approximately equivalent to the van der Waals energy. The zero electrostatic energy is due to the zero charge of the carboxyl group at acidic $\mathrm{pH}$. However, the surface charge of paclitaxel is also close to zero. Therefore, the electrostatic interaction between paclitaxel and $\mathrm{C60}$ is zero, and the van der Waals interaction is fragile. The weak interaction energies lead to a better release of the drug from C60 and are considered a decisive factor for the carrier, which can be very useful in drug release.

Fig.4 c. Shows the interactions between DOX and DMAA-TMC. The interesting point in the below diagram is that the interaction between the drug and the DMAA-TMC in an acidic state has positive electrostatic energy. This means that there is a repulsion between chitosan and the drug, and this is very effective in releasing the drug. Repulsion between DMAA-TMC and the drug causes a better release of the drug from the surface of the DMAA-TMC and C60. In fact, besides biocompatibility and hydrophilicity, DMAA-TMC plays a significant role in the mechanism of drug release in cancer tissue.

The figure 4 shows the interaction between DOX and DMAA-TMC. As can be seen from the figure, the van der Waals interaction is close to zero, but there is a significant negative electrostatic interaction between the drug and the DMAA-TMC. This interaction is due to the positive charge of DOX and the negative charge of DMAA-TMC at this $\mathrm{pH}$. The negative electrostatic energy indicates a strong attraction between the drug and the DMAA-TMC. DMAA-TMC is an essential aid in the absorption of the drug.

Fig.4 d. Shows the interaction energy between PAX and DMAA-TMCin an acidic state. As can be realized from the picture, the electrostatic interaction with van der Waals is zero between the drug and the 
chitosan, which contributes to drug release. Zero electrostatic energy is due to zero surface charge of PAX.

\section{Drug release mechanism}

As shown at Fig 6. Imine group includes a double bond between nitrogen and carbon atoms. The PAX/DOX-loaded nanomaterials via imine linkage could persevre drugs at physiological environment in the system and disassemble at acidic $\mathrm{pH}$ through the cleavage of imine bonds, which would face to immediately release DOX/PAX (50).

\section{Validation test}

To validate the computational procedure, a relevant recent article was chosen to reproduce a part of Gibbs free energy $(\Delta G)$ calculation (41). This article has studied the co-delivery of DOX/PAX by a nanotube-chitosan carrier. The average value of $\Delta \mathrm{G}$ is $-20.75 \mathrm{kcal} / \mathrm{mol}$ in the reference work for DOX adsorption on chitosan-nanotube (in acidic $\mathrm{pH}$ ) . The umbrella sampling simulation in GROMACS software was used to reproduce these data. Simulation results yielded a $\Delta \mathrm{G}$ value of $-21.64 \mathrm{kcal} / \mathrm{mol}$ that was near that of the reference study. These measurements can provide proof of this work and show consistency with previous works (41).

\section{Conclusion}

Combining biomaterials with nanoparticles is one of the essential functions of the smart drug delivery system. The results showed that finding new carriers with new compounds could be useful in improving the pharmacokinetic properties of pharmacodynamics and the therapeutic processes of drugs. Due to its significant implication in a variety of cancers, DOX is the focal point of drug research.

PAX is a natural source drug that develops taxel group drugs in the drug field. The molecular study of DOX-based carbonic $\mathrm{C} 60$ was the subject of this study, showing that $\mathrm{C} 60$ could be a suitable carrier for $\mathrm{pH}$-sensitive smart drug delivery by $\mathrm{pH}$ modification mechanism. In this computational work, the interactions between DOX and PAX with $\mathrm{C} 60$ were investigated at acidic and neutral pHs, along with the study of drug absorption and release at neutral and acidic $\mathrm{pHs}$, respectively. The exciting results show that the drug is highly absorbed in neutral pH (the normal pH of human blood), and its desired release occurs at acidic $\mathrm{pH}$ (the normal $\mathrm{pH}$ of cancerous tissue).

The presence of a biopolymer can affect the biocompatibility and biodegradability of the nanocarrier. Also, the effect of trimethyl chitosan as a suitable compound was investigated in this study. The results show that the combination of C60s and DMAA-TMCis sufficient for simultaneous absorption and release of DOX and PAX. As a suggestion for future work, this system can be tested both in the laboratory environment and in the tissues of living organisms.

In the physiological state $(\mathrm{pH}=7.4)$, the DMMA is attached to chitosan so that its interaction will result in increased absorption and hydrophilicity. On the other hand, this polymer is segregated from chitosan in 
the acidic state $(\mathrm{pH}=5.5)$, which improves the release of anticancer drugs. DMAA is a temperaturesensitive biopolymer that is susceptible to degradation in cancerous environments due to increased temperature. Furthermore, DMAA-TMCis a pH-sensitive polymer, thus the dual-sensitivity mechanism demonstrates the high capability of this novel nanocarrier. DMMA, as an amphiphile polymer, has many advantages in drug development, including better nanocarrier aggregation, decreased toxicity, and reduced nanoparticle size for cell penetration.

On the other hand, the electrostatic interactions of DOX and van der Waals interactions of PAX with C60s decrease and the drugs are more easily released in the cancerous environment. Also, the pharmacokinetic problems of PAX can be underestimated due to improved solubility and penetration, as well as reduced cardiac toxicity of DOX owing to its smart release. The previous simulations in similar articles were repeated as a validation test.

\section{Declarations}

\section{Ethics approval and consent to participate}

Not applicable

\section{Consent for publication}

Not applicable

\section{Availability of data and materials}

Not applicable

\section{Competing interests}

The authors declare that they have no competing interests

\section{Funding}

Not applicable

\section{Authors' contributions}

Ehsan Alimohammadi; supervision, writing , review and editing 
Reza Maleki; conceptualization, methodology, software, validation

Hossein Akbariakiabad;writing , original draft

Mohammad Dahri; conceptualization, visualization, data curation

Acknowledgements

The authors wish to acknowledge Fatemeh Alimohammadi for her support in Review \& Editing.We would also like to show our appreciation to Mohammad Mahdi Nematollahi and Dr.kalhor for sharing in conceptualization and editing of this work.

Author informations

Ehsan Alimohammadi

https://orcid.org/0000-0002-6373-6248 email: hafez125@gmail.com

Reza Maleki

https://orcid.org/0000-0002-5169-5848 email: rezamaleki96@gmail.com

Hossein Akbarialiabad

https://orcid.org/0000-0003-2018-6378 email:HosseinAkbarialiabad7575@gmail.com

Mohammad Dahri

https://orcid.org/0000-0003-1776-7243 email: mdahri1996@gmail.com

\section{Abbreviations}




\begin{tabular}{ll}
\hline Abbreviations & Full form \\
\hline C60 & Fullerene \\
CML & Chronic myelogenous leukemia \\
DDS & Drug delivery systems \\
DMAA-TMC & Dimethyl acryl amid- trimethyl chitosan \\
DOX & Doxorubicin \\
Fig & Figure \\
GROMACS & Groningen machine for chemical simulations \\
OPLS-aa & Optimized Potentials for Liquid Simulations-all atom \\
PAX & Paclitaxel \\
Rg & Radius of Gyration \\
\hline
\end{tabular}

\section{References}

1. McPherson K, Steel C, Dixon J. ABC of breast diseases: breast cancer-epidemiology, risk factors, and genetics. BMJ: British Medical Journal. 2000;321(7261):624.

2. Stämpfli MR, Anderson GP. How cigarette smoke skews immune responses to promote infection, lung disease and cancer. Nature Reviews Immunology. 2009;9(5):377-84.

3. O'keefe SJ. Diet, microorganisms and their metabolites, and colon cancer. Nature reviews Gastroenterology \& hepatology. 2016;13(12):691.

4. Galluzzi L, Buqué A, Kepp O, Zitvogel L, Kroemer G. Immunogenic cell death in cancer and infectious disease. Nature Reviews Immunology. 2017;17(2):97.

5. Havas M. When theory and observation collide: Can non-ionizing radiation cause cancer? Environmental Pollution. 2017;221:501-5.

6. Burgess A, Chia KM, Haupt S, Thomas D, Haupt Y, Lim E. Clinical overview of MDM2/X-targeted therapies. Frontiers in oncology. 2016;6:7.

7. Delbridge A, Strasser A. The BCL-2 protein family, BH3-mimetics and cancer therapy. Cell Death \& Differentiation. 2015;22(7):1071-80.

8. Haupt S, Raghu D, Haupt Y. Mutant p53 drives cancer by subverting multiple tumor suppression pathways. Frontiers in oncology. 2016;6:12.

9. Jin X, Ding D, Yan Y, Li H, Wang B, Ma L, et al. Phosphorylated RB promotes cancer immunity by inhibiting NF-KB activation and PD-L1 expression. Molecular cell. 2019;73(1):22-35. e6.

10. Breugom AJ, Swets M, Bosset J-F, Collette L, Sainato A, Cionini L, et al. Adjuvant chemotherapy after preoperative (chemo) radiotherapy and surgery for patients with rectal cancer: a systematic review 
and meta-analysis of individual patient data. The lancet oncology. 2015;16(2):200-7.

11. Alvarez-Lorenzo C, Concheiro A. Smart drug delivery systems: from fundamentals to the clinic. Chemical Communications. 2014;50(58):7743-65.

12. Kayser O, Lemke A, Hernandez-Trejo N. The impact of nanobiotechnology on the development of new drug delivery systems. Current pharmaceutical biotechnology. 2005;6(1):3-5.

13. Ramasamy T, Ruttala HB, Gupta B, Poudel BK, Choi H-G, Yong CS, et al. Smart chemistry-based nanosized drug delivery systems for systemic applications: a comprehensive review. Journal of Controlled Release. 2017;258:226-53.

14. Wang H, Liu X, Wang Y, Chen Y, Jin Q, Ji J. Doxorubicin conjugated phospholipid prodrugs as smart nanomedicine platforms for cancer therapy. Journal of Materials Chemistry B. 2015;3(16):3297-305.

15. Rezvantalab S, Moraveji M, Khedri M, Maleki R. An Insight into The Role of Riboflavin Ligand on the Self-assembly of Poly (lactic-co-glycolic acid)-based Nanoparticles, A Molecular Simulation and Experimental Approach. Soft Matter. 2020.

16. Rezaian M, Maleki R, Dahri Dahroud M, Alamdari A, Alimohammadi M. pH-Sensitive CoAdsorption/Release of Doxorubicin and Paclitaxel by Carbon Nanotube, Fullerene, and Graphene Oxide in Combination with N-isopropylacrylamide: A Molecular Dynamics Study. Biomolecules. 2018;8(4):127.

17. Tharkar-Promod S, Johnson DP, Bennett SE, Dennis EM, Banowsky BG, Jones SS, et al. HDAC1, 2 inhibition and doxorubicin impair Mre11-dependent DNA repair and DISC to override BCR-ABL1driven DSB repair in Philadelphia chromosome-positive B-cell precursor acute lymphoblastic leukemia. Leukemia. 2018;32(1):49-60.

18. Renu K, Abilash V, PB TP, Arunachalam S. Molecular mechanism of doxorubicin-induced cardiomyopathy-An update. European journal of pharmacology. 2018;818:241-53.

19. Pugazhendhi A, Edison TNJI, Velmurugan BK, Jacob JA, Karuppusamy I. Toxicity of Doxorubicin (Dox) to different experimental organ systems. Life sciences. 2018;200:26-30.

20. Halim VA, García-Santisteban I, Warmerdam DO, Van den Broek B, Heck AJ, Mohammed S, et al. Doxorubicin-induced DNA damage causes extensive ubiquitination of ribosomal proteins associated with a decrease in protein translation. Molecular \& Cellular Proteomics. 2018;17(12):2297-308.

21. Wang Y, Xu Z. Interaction mechanism of doxorubicin and SWCNT: protonation and diameter effects on drug loading and releasing. RSC advances. 2016;6(1):314-22.

22. Zambrano-Estrada X, Landaverde-Quiroz B, Dueñas-Bocanegra AA, De Paz-Campos MA, HernándezAlberto $\mathrm{G}$, Solorio-Perusquia B, et al. Molecular iodine/doxorubicin neoadjuvant treatment impair invasive capacity and attenuate side effect in canine mammary cancer. BMC veterinary research. 2018;14(1):87.

23. Zheng Y, Ji X, Yu B, Ji K, Gallo D, Csizmadia E, et al. Enrichment-triggered prodrug activation demonstrated through mitochondria-targeted delivery of doxorubicin and carbon monoxide. Nature chemistry. 2018;10(7):787-94. 
24. Leung JC, Cassimeris L. Reorganization of paclitaxel-stabilized microtubule arrays at mitotic entry: roles of depolymerizing kinesins and severing proteins. Cancer biology \& therapy. 2019;20(10):133747.

25. Khongkow P, Gomes A, Gong C, Man E, Tsang JW, Zhao F, et al. Paclitaxel targets FOXM1 to regulate KIF20A in mitotic catastrophe and breast cancer paclitaxel resistance. Oncogene. 2016;35(8):9901002.

26. Reddy D, Choonara YE, Kumar P, Govender M, Indermun S, du Toit LC, et al. In vivo evaluation of an Ultra-fast Disintegrating Wafer matrix: A molecular simulation approach to the ora-mucoadhesivity. Journal of Drug Delivery Science and Technology. 2017;37:123-33.

27. Liang W, Zhuo Y, Xiong C, Zheng Y, Chai Y, Yuan R. Ultrasensitive cytosensor based on self-enhanced electrochemiluminescent ruthenium-silica composite nanoparticles for efficient drug screening with cell apoptosis monitoring. Analytical chemistry. 2015;87(24):12363-71.

28. Huttunen KM, Gynther M, Huttunen J, Puris E, Spicer JA, Denny WA. A selective and slowly reversible inhibitor of I-type amino acid transporter 1 (LAT1) potentiates Antiproliferative drug efficacy in Cancer cells. Journal of medicinal chemistry. 2016;59(12):5740-51.

29. Lavan AH, O'Mahony D, Buckley M, O'Mahony D, Gallagher P. Adverse drug reactions in an oncological population: prevalence, predictability, and preventability. The oncologist. 2019;24(9):e968-e77.

30. Paliwal R, Paliwal SR, Kenwat R, Kurmi BD, Sahu MK. Solid lipid nanoparticles: a review on recent perspectives and patents. Expert Opinion on Therapeutic Patents. 2020:1-16.

31. Skipa TA, Koltover VK. Fullerene Trend in Biomedicine: Expectations and Reality. Neurosci Res. 2019;86(16):3622-34.

32. Li Z, Liu Z, Sun H, Gao C. Superstructured Assembly of Nanocarbons: Fullerenes, Nanotubes, and Graphene. Chemical Reviews. 2015;115(15):7046-117.

33. Castro E, Garcia AH, Zavala G, Echegoyen L. Fullerenes in biology and medicine. Journal of Materials Chemistry B. 2017;5(32):6523-35.

34. Asghari F, Khademi R, Esmaeili Ranjbar F, Veisi Malekshahi Z, Faridi Majidi R. Application of Nanotechnology in Targeting of Cancer Stem Cells: A Review. Int J Stem Cells. 2019;12(2):227-39.

35. Xiao L, Huang R, Zhang Y, Li T, Dai J, Nannapuneni N, et al. A New Formyl Peptide Receptor-1 Antagonist Conjugated Fullerene Nanoparticle for Targeted Treatment of Degenerative Disc Diseases. ACS Applied Materials \& Interfaces. 2019;11(42):38405-16.

36. García-Simón C, Monferrer A, Garcia-Borràs M, Imaz I, Maspoch D, Costas M, et al. Size-selective encapsulation of $\mathrm{C} 60$ and $\mathrm{C} 60$-derivatives within an adaptable naphthalene-based tetragonal prismatic supramolecular nanocapsule. Chemical Communications. 2019;55(6):798-801.

37. Pérez-Calixto MP, Ortega A, Garcia-Uriostegui L, Burillo G. Synthesis and characterization of Nvinylcaprolactam/N,N-dimethylacrylamide grafted onto chitosan networks by gamma radiation. Radiation Physics and Chemistry. 2016;119:228-35. 
38. Maleki R, Afrouzi HH, Hosseini M, Toghraie D, Piranfar A, Rostami S. pH-sensitive loading/releasing of doxorubicin using single-walled carbon nanotube and multi-walled carbon nanotube: A molecular dynamics study. Computer methods and programs in biomedicine. 2020;186:105210.

39. Sahooli M, Sabbaghi S, Maleki R, Nematollahi MM. Thermal conductivity of Water-based nanofluids: Prediction and comparison of models using machine learning \%J International Journal of Nano Dimension. 2014;5(1):47-55.

40. Maleki R, Khoshoei A, Ghasemy E, Rashidi A. Molecular insight into the smart functionalized TMCFullerene nanocarrier in the $\mathrm{pH}$-responsive adsorption and release of anti-cancer drugs. Journal of Molecular Graphics and Modelling. 2020;100:107660.

41. Karnati KR, Wang Y. Understanding the co-loading and releasing of doxorubicin and paclitaxel using chitosan functionalized single-walled carbon nanotubes by molecular dynamics simulations. Physical Chemistry Chemical Physics. 2018;20(14):9389-400.

42. Khoshoei A, Ghasemy E, Poustchi F, Shahbazi M-A, Maleki R. Engineering the pH-sensitivity of the graphene and carbon nanotube based nanomedicines in smart cancer therapy by grafting trimetyl Chitosan. Pharmaceutical Research. 2020;37(8):1-13.

43. Tuszynski JA, Winter $P$, White $D$, Tseng $C-Y$, Sahu KK, Gentile F, et al. Mathematical and computational modeling in biology at multiple scales. Theoretical Biology and Medical Modelling. 2014;11(1):52.

44. Alimohammadi E, Khedri M, Jahromi AM, Maleki R, Rezaian M. Graphene-Based Nanoparticles as Potential Treatment Options for Parkinson's Disease: A Molecular Dynamics Study. International Journal of Nanomedicine. 2020;15:6887.

45. Jain S, Ancheria RK, Shrivastava S, Soni SL, Sharma MJAJoPR, Development. An Overview of Nanogel-Novel Drug Delivery System. 2019;7(2):47-55.

46. Feinstein WP, Brylinski M. Calculating an optimal box size for ligand docking and virtual screening against experimental and predicted binding pockets. Journal of Cheminformatics. 2015;7(1):18.

47. Dunnill CJ, Al-Tameemi W, Collett A, Haslam IS, Georgopoulos NTJTo. A Clinical and Biological Guide for Understanding Chemotherapy-Induced Alopecia and Its Prevention. 2018;23(1):84-96.

48. Lobanov MY, Bogatyreva NS, Galzitskaya OV. Radius of gyration as an indicator of protein structure compactness. Molecular Biology. 2008;42(4):623-8.

49. He X, Gao W, Xie L, Li B, Zhang Q, Lei S, et al. Wafer-scale monodomain films of spontaneously aligned single-walled carbon nanotubes. Nature Nanotechnology. 2016;11(7):633-8.

50. Tao Y, Liu S, Zhang Y, Chi Z, Xu J. A pH-responsive polymer based on dynamic imine bonds as a drug delivery material with pseudo target release behavior. Polymer Chemistry. 2018;9(7):878-84.

\section{Figures}




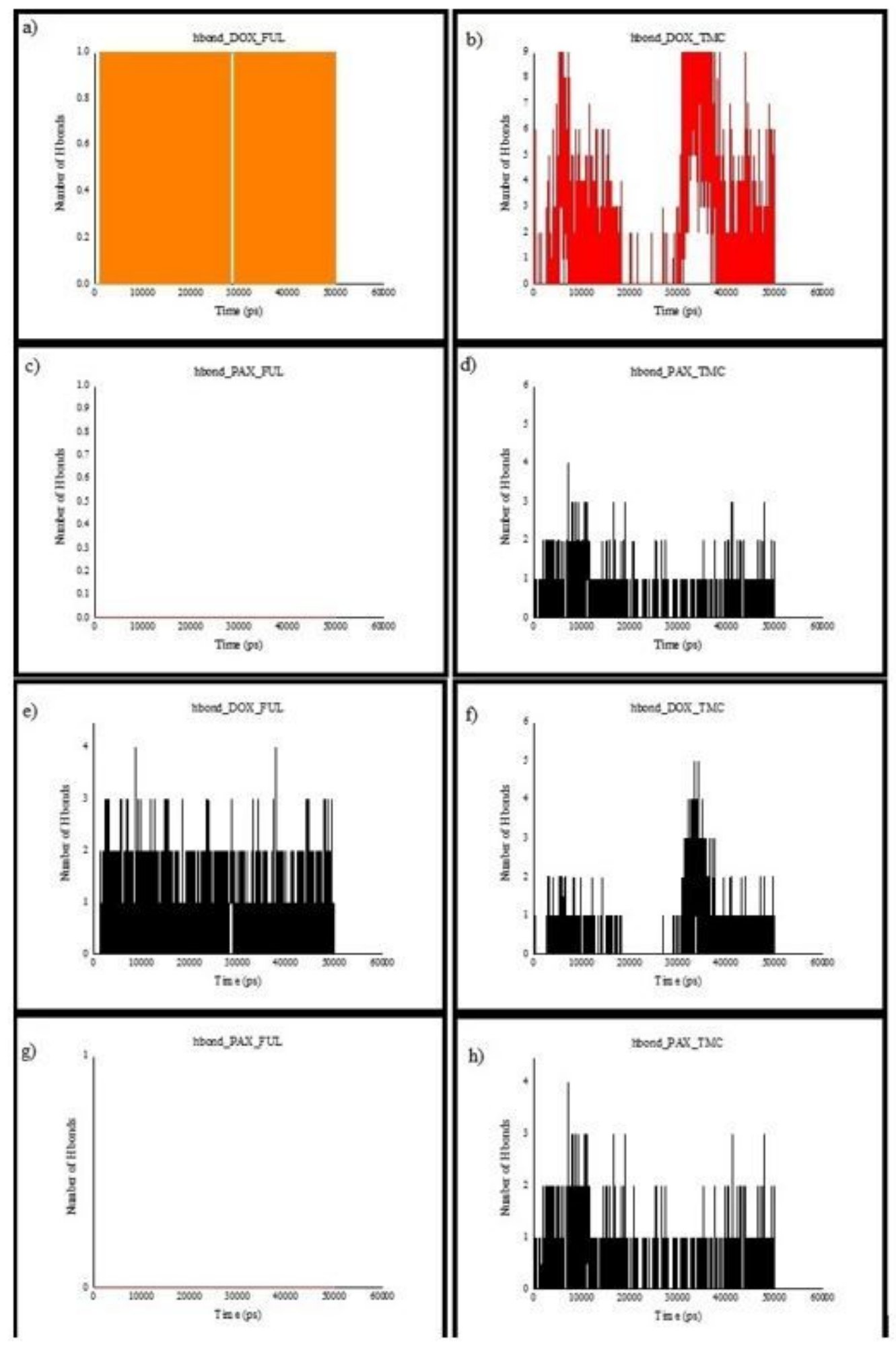

Figure 1

Interaction hydrogen bonds a) The number of hydrogen bonds between DOX and C60 versus time in neutral $\mathrm{pH} \mathrm{b}$ ) The number of hydrogen bonds between DOX and DMAA-TMC versus time in neutral $\mathrm{pH}$ c) The number of hydrogen bonds between PAX and $\mathrm{C} 60$ versus time in neutral $\mathrm{pH} d$ ) The number of hydrogen bonds between DMAA-TMC and PAX versus time in neutral $\mathrm{pH}$ e) The number of hydrogen bonds between $\mathrm{DOX}$ and $\mathrm{C} 60$ versus time in acidic $\mathrm{pH}$ f) The number of hydrogen bonds between DOX 
and DMAA-TMC versus time in acidic $\mathrm{pH} g$ ) The number of hydrogen bonds between PAX and $\mathrm{C} 60$ versus time in acidic $\mathrm{pH} h$ ) The number of hydrogen bonds between PAX and DMAA-TMC versus time in acidic $\mathrm{pH}$
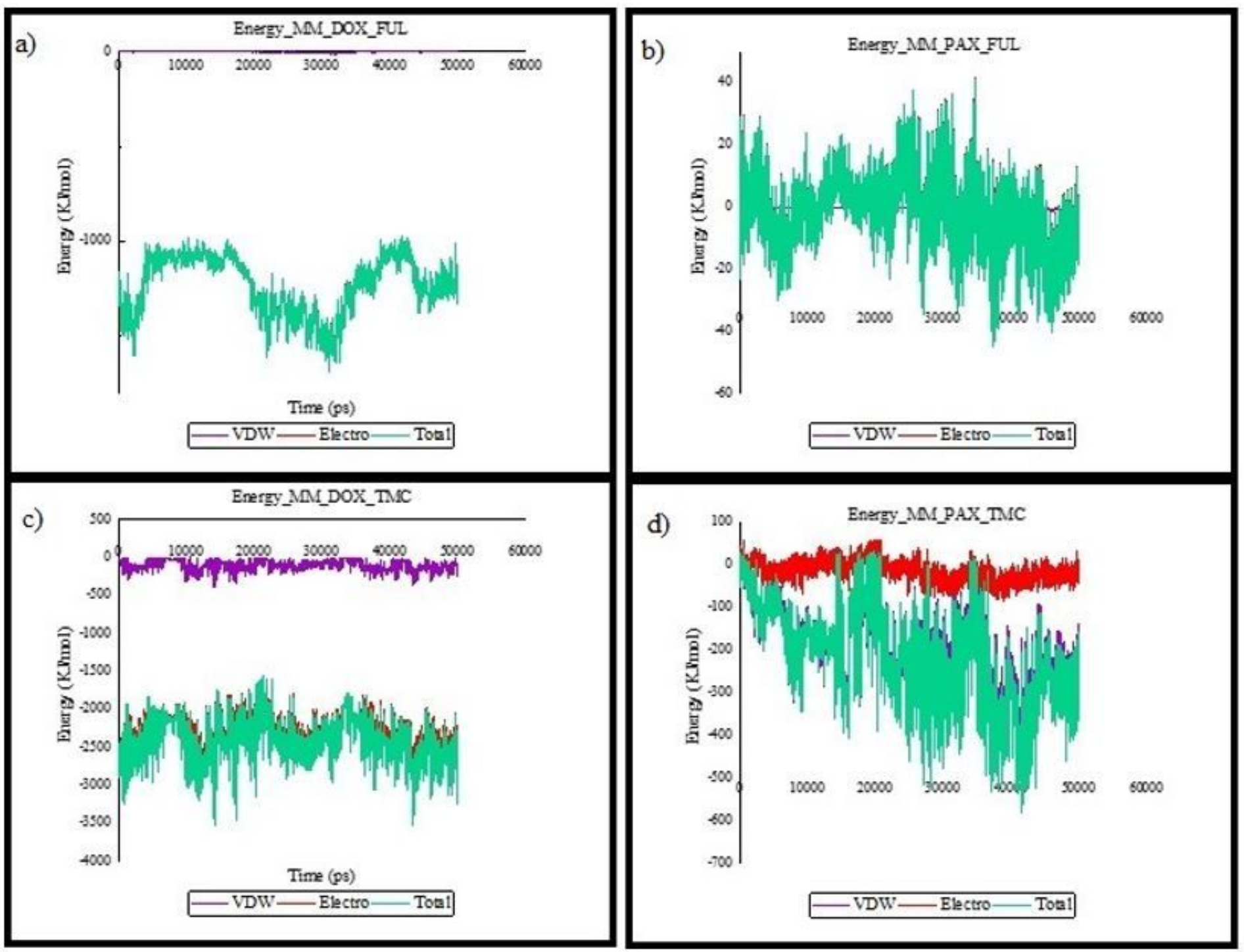

Figure 2

Interaction energies between $\mathrm{C} 60$ and Drugs, at pH=7.4 a: DOX-C60 b: PAX-C60 c:DOX-TMC d: PAX-TMC 


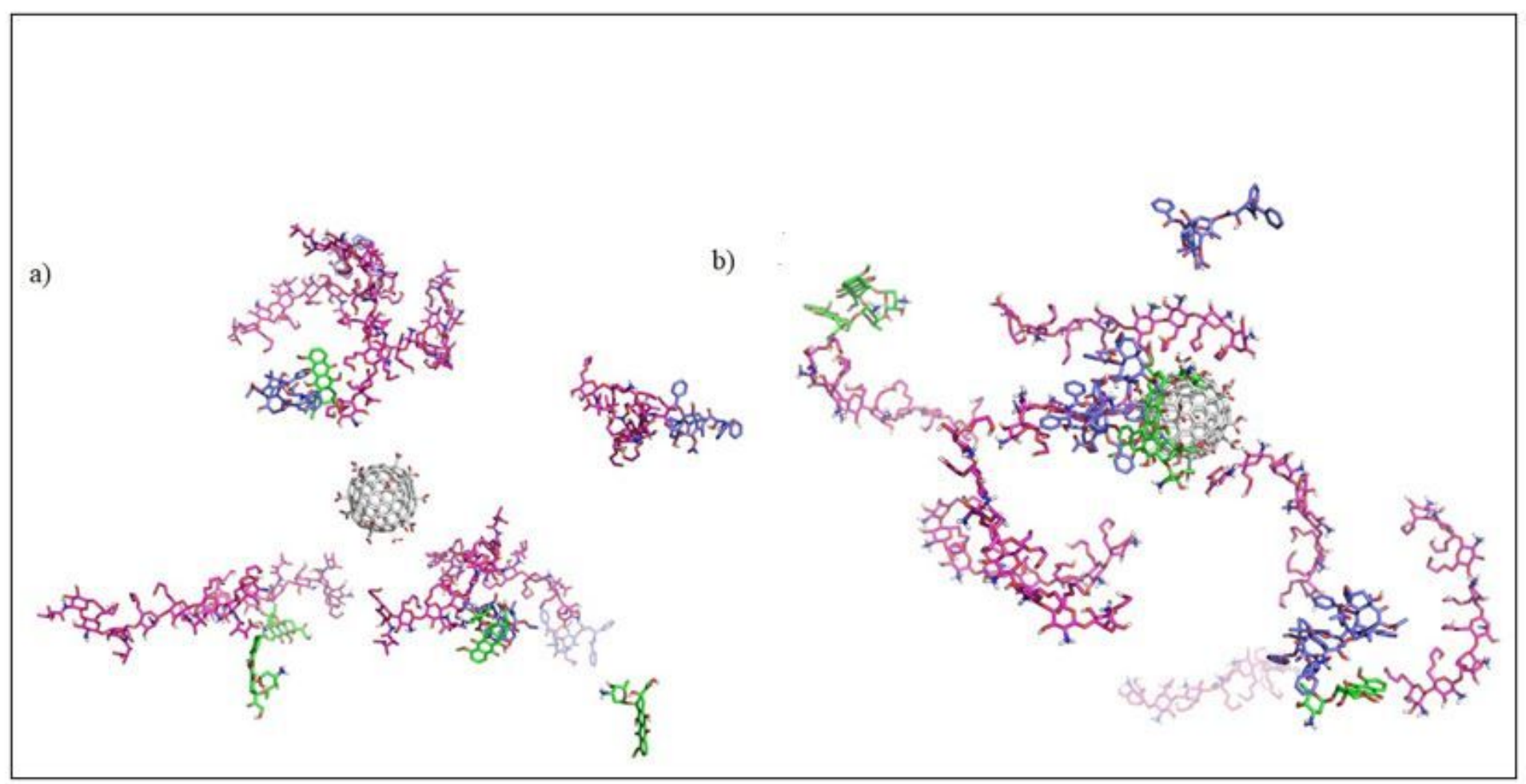

Figure 3

Molecular simulation of DMAA-TMC functionalized C60; $a: t=0 \mathrm{~ns}, \mathrm{~b}: \mathrm{t}=50 \mathrm{~ns}$ at $\mathrm{pH}=7.4$ 


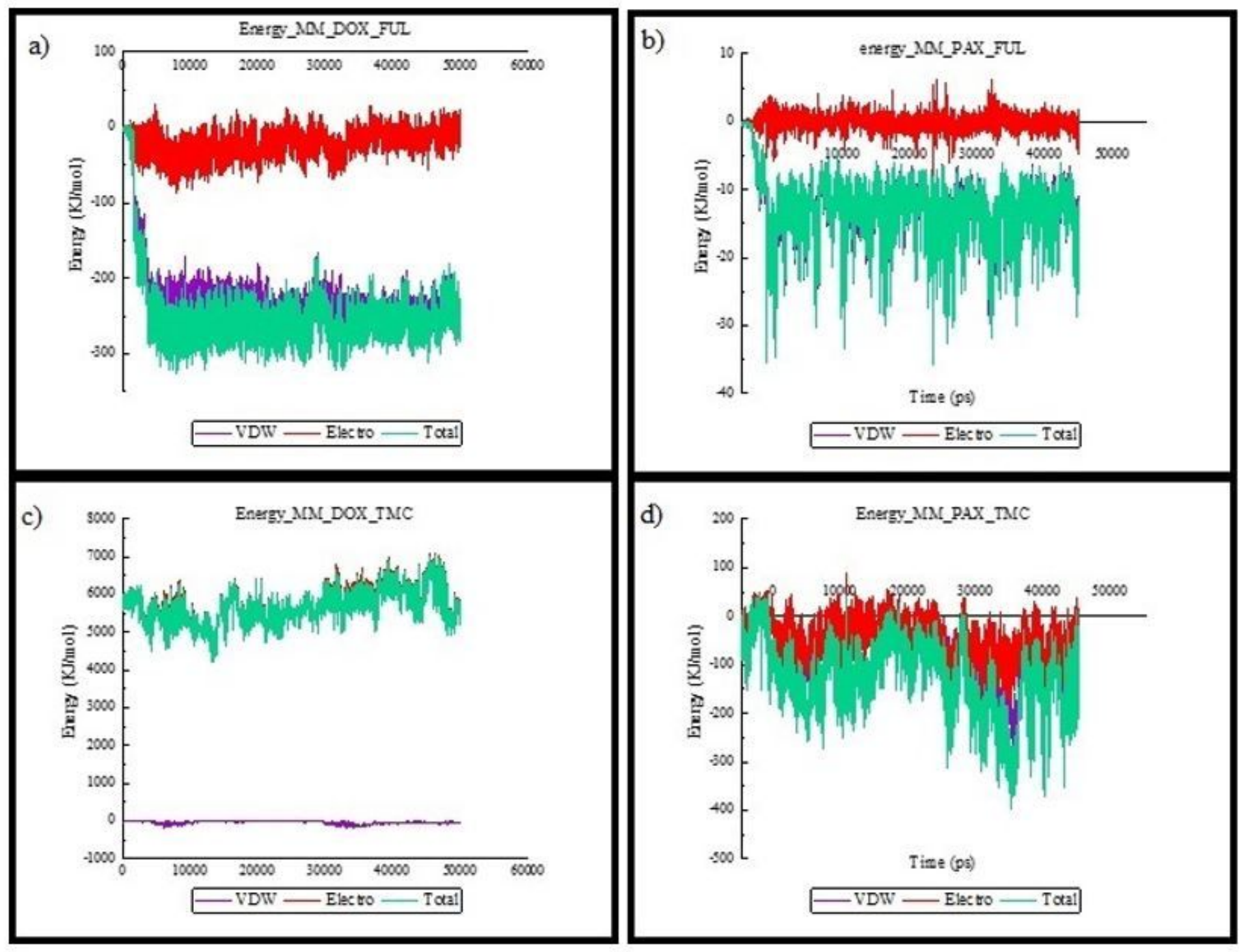

Figure 4

Interaction energies between $\mathrm{C} 60$ and Drugs, at pH=5.5 a: DOX-C60 b: PAX-C60 c:DOX-TMC d: PAX-TMC 


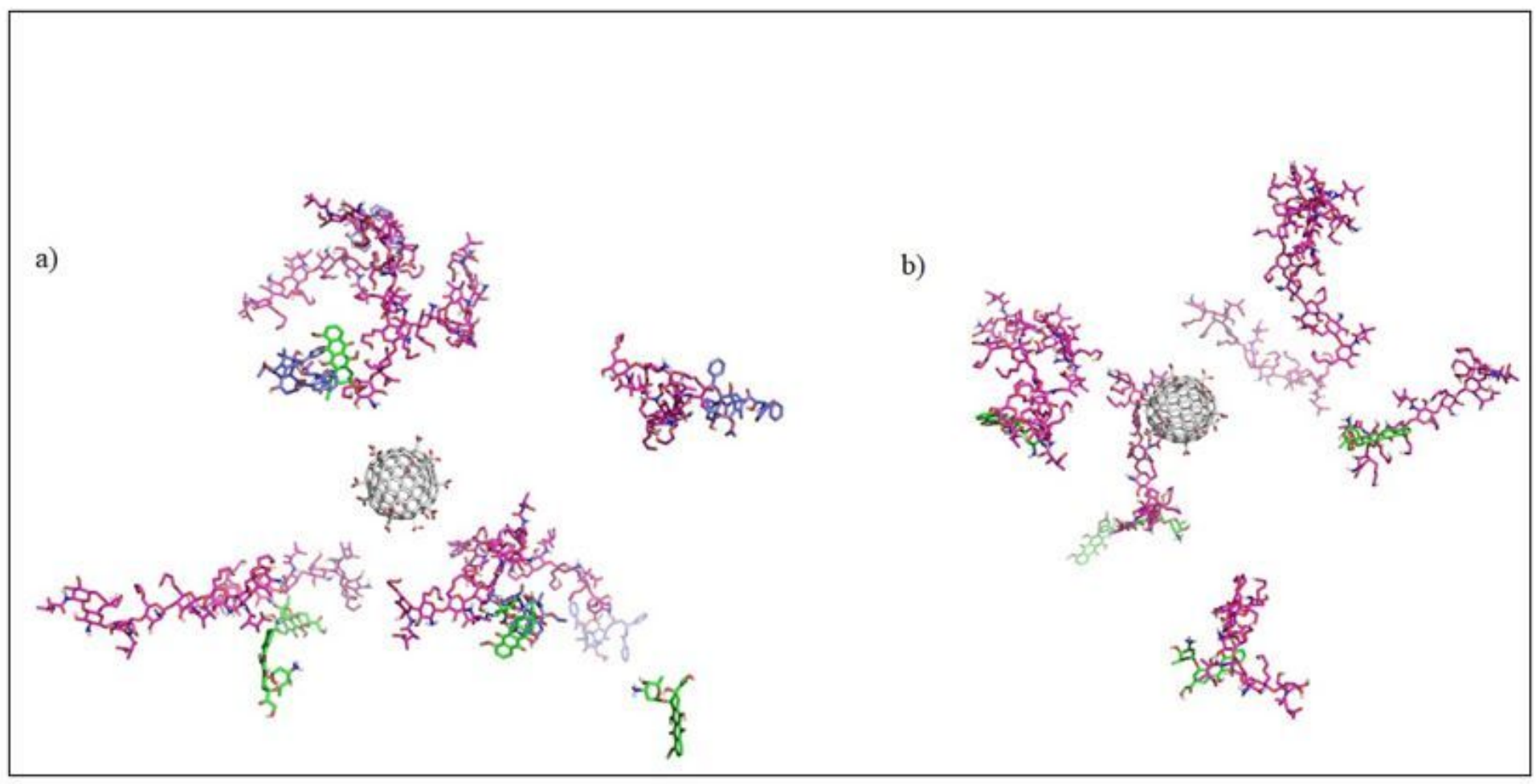

\section{Figure 5}

Molecular simulation of DMAA-TMC functionalized C60; a: loaded, b: unloaded with $\mathrm{PAX}$ at $\mathrm{pH}=5.5$ 


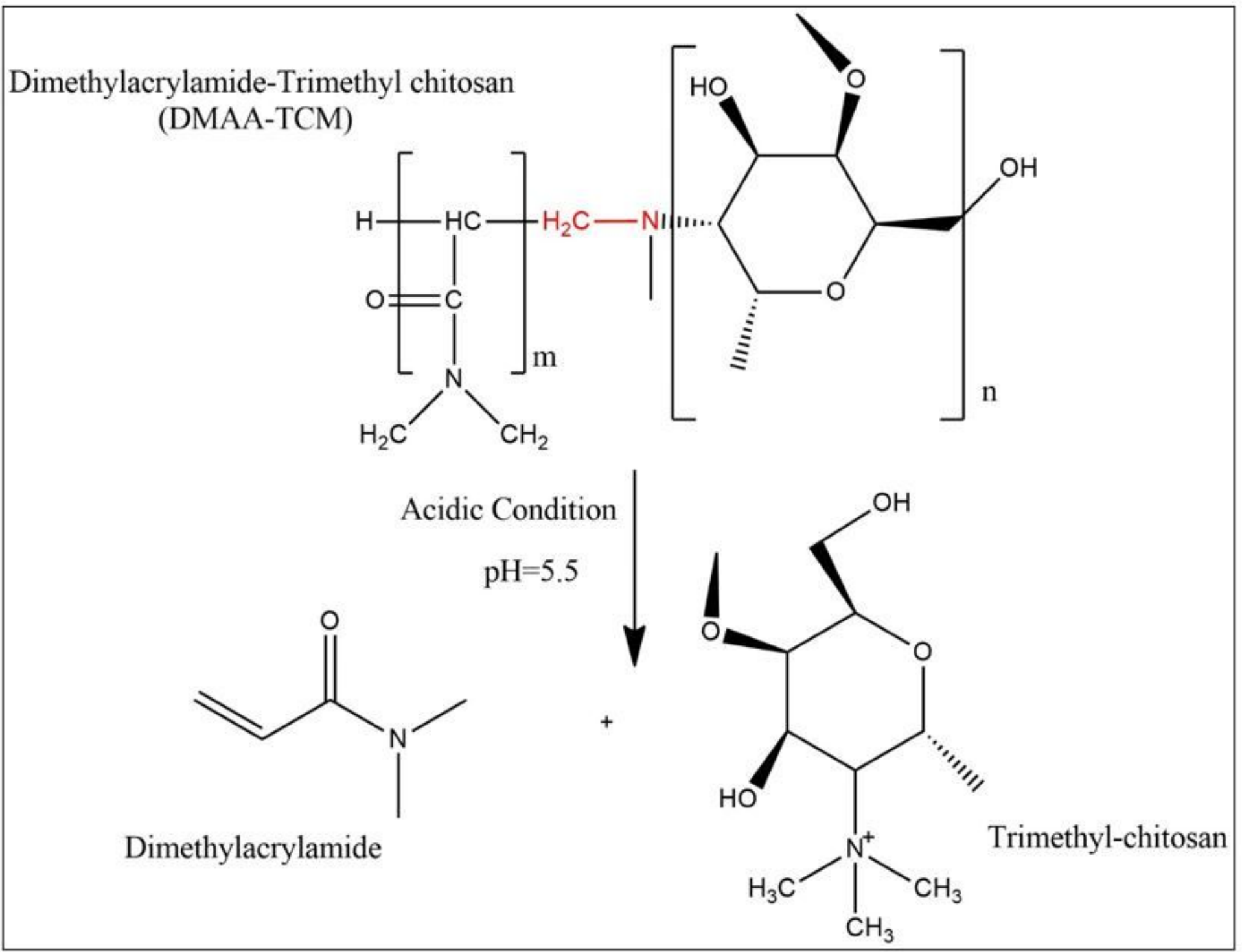

Figure 6

DMAA and TMC dissamlation mechanism at Acidic $\mathrm{pH}$ 